\title{
Modulation Transfer Function Obtained from Image Structures
}

\author{
Fang Lei, Hans Tiziani
}

Institut für Technische Optik, Universität Stuttgart

\section{Summary}

A new and relatively simple method for edge gradient analysis (EGA) has been developed to determine the modulation transfer function (MTF) of photogrammetrical systems. The MTF can be calculated as the ratio of the amplitude of those frequencies present in the original edge to the amplitude of the same frequency in the scanned edge image. Thus a differentiation of the edge scan - as usually implemented - is not necessary. In order to avoid a division by zero we multiplied the edge images by the so called Hanning function before performing the Fourier analysis. From the point of noise sensitivity the method using a Hanning window is advantageous in comparison to the edge differentiation method. The edge gradient analysis method and the grating pattern method are compared. Artificial edge, natural roof edge, and grating patterns were used to determine the quality of the photogrammetrical system. Good agreement of all MTF measurements was found. Furthermore, the resolution found from MTF curves agreed well with the resolution obtained from threebar targets. Generally, the MTF curves obtained from patterns in the flight direction were lower than the MTF's perpendicular to the flight direction due to airplane movement. The influence of linear image motion and its compensation was examined and is discussed. Furthermore, the longitudinal motion of the object as defect of focus is studied analytically and experimentally for a close range.

\section{Introduction}

In photogrammetry the image quality of photographs is of great importance. The optical transfer function (OTF) and especially the modulation transfer function (MTF) are useful techniques for 
measuring the image quality of photographs. The MTF is the result of the contributions of the links in the image transfer forming chain, namely, the camera, the film, image motion (in systems without forward motion compensation, FMC), vibratins, and the atmosphere /1/.

In this paper, a new method for edge gradient analysis (EGA), using a Hanning window, is developed and discussed. A comparison is made with the other methods to measure the MTF of aerial survey lens systems from the image structures. The measured MTF curves are given showing the reliability of different methods. Furthermore, the influences of linear image motion and focusing defect on image quality is examined.

\section{Methods for Determining the MTF from Image Structures}

The edge gradient analysis (EGA) is a technique for obtaining the MTF from edge images recorded on photographic film, especially useful for evaluation of images not containing targets. During the past 20 years different calculation methods for EGA have been developed $/ 2-6 /$. One method, based on the differentiation of the edge, has often been used for MTF determination. The OTF is determined from the Fourier transform of the spread function, obtained from differentiating the edge image. It can be called an edge differentiation method. We have developed a new and relatively simple method for EGA /6/. The method is based on the Fourier spectrum amplitude ratio between the real and an ideal edge image. It can be called an edge spectrum ratio method. From a purely mathematical analysis both methods are equivalent. However for practical applications they have to be treated differently.

In the edge spectrum ratio method, the MTF is obtained by dividing the spatial frequency spectrum of an ideal edge. From the definition of the MTF, it follows that

$$
T(R)=|D(R)|=\left|\frac{k^{\prime}(R)}{k(R)}\right|
$$


where $k(R), k^{\prime}(R)$, and $D(R)$ are the Fourier-transforms of an ideal edge, a scanned edge, and the line spread funktion respectively.

The division of the direct numerical Fourier-transforms of the edge in the image and object space leads to a problem because infinitely expanded edges must be limited by a multiplication with a rectangular function (window), leading to zero amplitudes at even harmonic frequencies in the spectrum of the ideal edge. To avoid a division by zero, the rectangular function must be replaced by another window function. We found that the so called Hanning function is a very suitable window function.

The Hanning function can be written as /7/

$$
H(u)= \begin{cases}1 / 2-1 / 2 \cos (2 \pi u / L) & 0 \leqslant u \leqslant L \\ 0 & u<0, u>L\end{cases}
$$

with the length $\mathrm{L}$ of the limited edge.

A smoothed curve is obtained from the spectrum of an edge image multiplied by the Hanning function, which is in good agreement with the spectrum's envelope. Zero amplitudes are no longer present when using the modified edge spectrum ratio method. With a Hanning window the equation (1) can be rewritten as

$$
T(R)=\left|\frac{k_{H}^{\prime}(R)}{k_{H}(R)}\right|
$$

where $k_{H}^{\prime}(R)$ and $k_{H}(R)$ are the Fourier-transforms of the Hanning function multiplied by a scanned edge and an ideal edge respectively.

Figure I shows schematically the procedure for the MTF determination. Before applying the Fourier-transformation, both scanned 


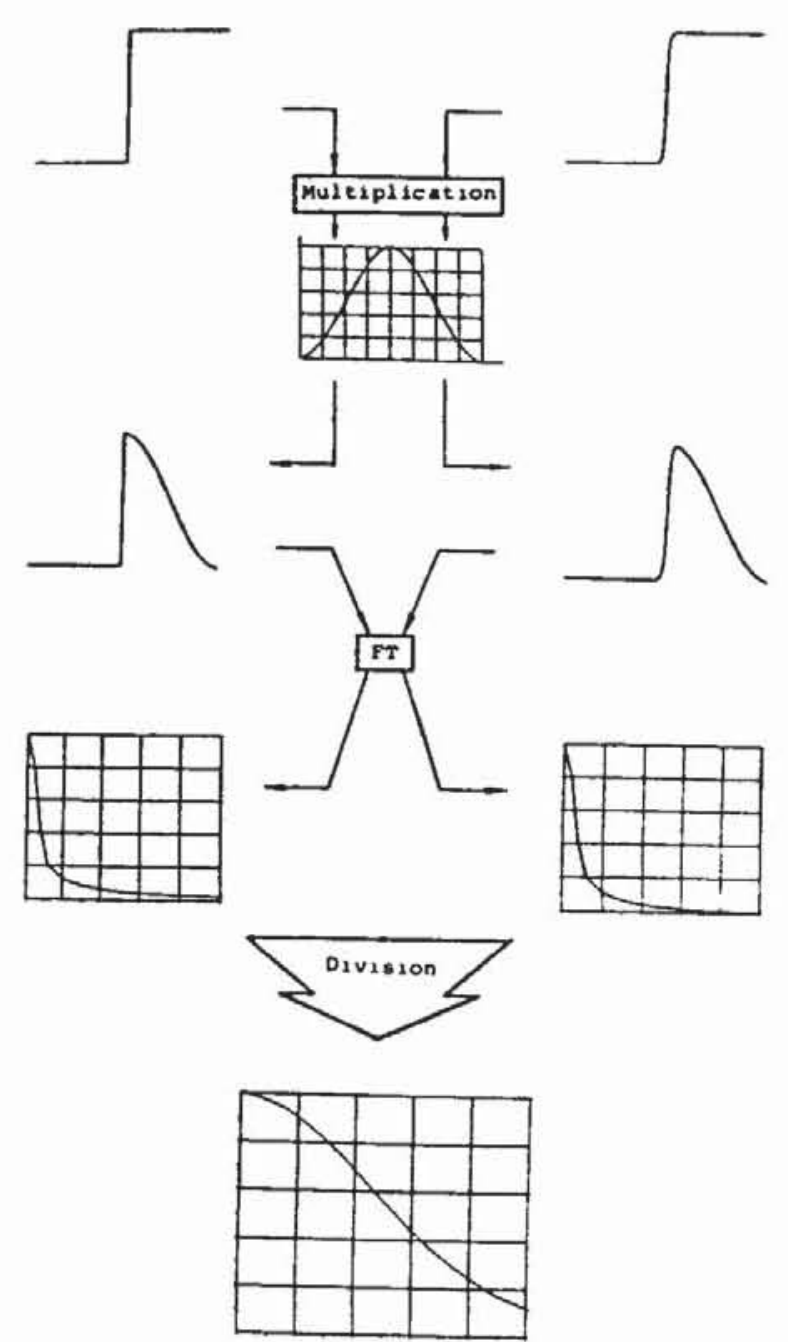

Fig.1 Diagram to calculate the MTF using the Hanning function and ideal edges must be multiplied by the Hanning function. By the introduction of the Hanning function, noise on top of an edge image can also be suppressed. The edge spectrum ratio method has been compared with the edge differentiation method. From the point of noise sensitivity the edge spectrum ratio method using a Hanning window is advantageous.

For comparison with EGA, a variable spatial frequency grating pattern was used for the determination of the MTF. The contrast of the photographed pattern decreases due to diffraction and different disturbances with increasing spatial frequency. Using the convolution, the imaging process can be simulated with a computer. The intensity distribution of the grating pattern is convoluted with a compu-

ter simulated model of the spread function and the result should compare with that of the scanned image of the grating $/ 8 /$. The MTF is calculated as the absolute value of the Fourier-transform of the chosen spread function.

3. Comparison between the MTF's obtained from an Edge Image and a Grating Pattern

A pair of artificial edge and grating patterns was oriented in the flight direction, and another pair was oriented orthogonally to it. Two suitable natural roof edges were used to compare the artificial edge. The test pattern images and the natural edge 
images were scanned with a computer controlled microdensitometer and the measurements were evaluated by a computer.

Figure 2 shows the MTF's obtained from grating patterns and from artificial and natural edges both in the flight direction (a) and perpendicular to the flight direction (b). It should be noted that there is little difference in the MTF of a natural and an artificial edge. We found good agreement of the results obtained with different test patterns.

For an additional test of the MTF, we examined three-bar patterns with different spatial frequencies. They were put close to the grating and edge patterns in order to determine resolution limits. The spatial frequencies at the intersection of the MTF, for well-defined artificial edges, and the threshold curves compare well with the resolution limits obtained from the three-bar pattern in the flight direction and perpendicular to it, respec-
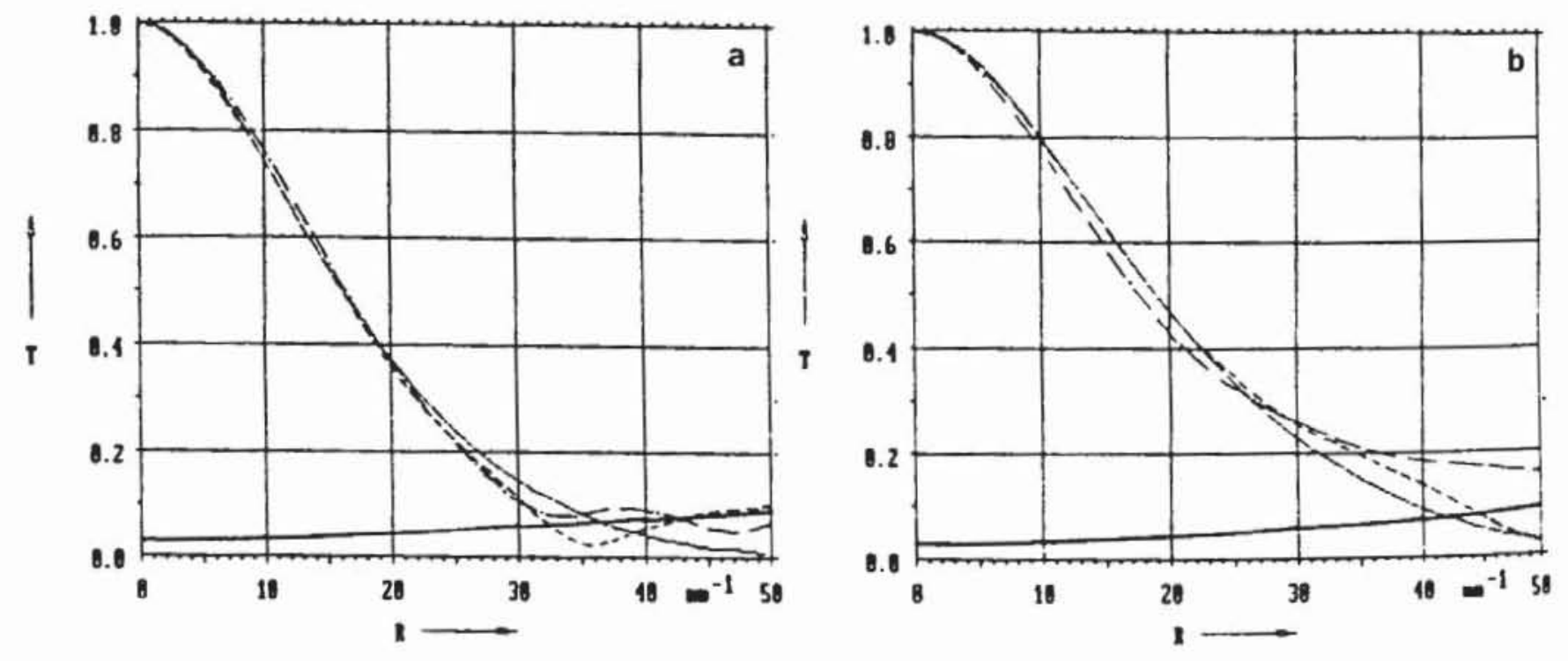

Fig.2 (a): MTF of the test patterns in flight direction. (b): MTF of the test patterns perpendicular to the flight direction.

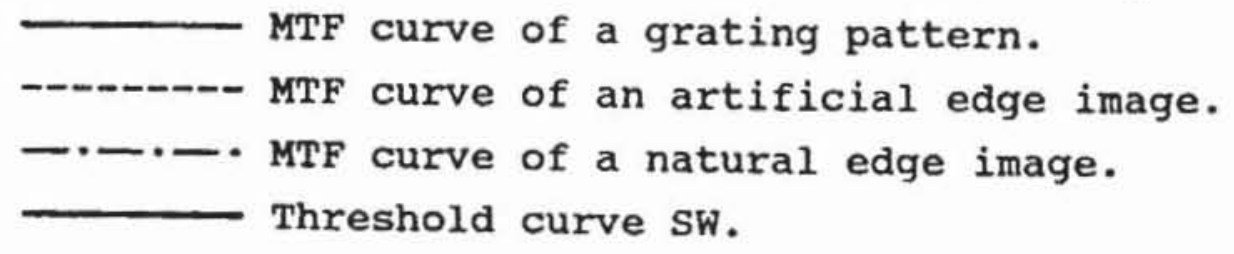


tively. This comparison proves the reliability of the different methods for the determination of image quality.

\section{Influence of Linear Image Motion on Image Quality}

There are different types of image motions such as linear, parabolic, sinusoidal, and random $/ 1 /$. This paper deals only with linear motion. The quality of airborne photographs can be improved by applying forward motion compensation (FMC). The photographs thus far referred to in this paper were obtained without using FMC, and this explains why in Figure 2 the MTF's of test patterns oriented in the flight direction are worse than those perpendicular to it. From the difference of the MTF's in the two directions, the image motion can be calculated. It will be compared with the theoretical image motion.

The image displacement during exposure time $T$ is

$$
a=\operatorname{vTf}^{\prime} / \mathrm{H}
$$

where $v$ is the speed of the airplane, $f^{\prime}$ is the focal length of the objective, and $\mathrm{H}$ is the altitude of the airplane. The spread function corresponding to the movement is a rectangular function of width a. The corresponding MTF is its Fourier transform

$$
\mathrm{MTF}_{\mathrm{B}}=\mathrm{a} \frac{\sin \pi \mathrm{Ra}}{\pi \mathrm{Ra}}
$$

where $R$ is the spatial frequency and $a$ is the image motion. This is a sinc-function. If the MTF is measured perpendicular to the flight direction and multiplied by this sinc-function, the result measured in the flight direction should be obtained providing the signal-to-noise ratio is not to bad. According to linear response theory, the $\mathrm{MTF}_{\mathrm{F}}$ in flight direction can be written as

$$
\mathrm{MTF}_{\mathrm{F}}=\mathrm{MTF}_{\mathrm{B}} \cdot \mathrm{MTF}_{\mathrm{P}}
$$


where $\mathrm{MTF}_{\mathrm{P}}$ is perpendicular to the flight direction and $\mathrm{MTF}_{\mathrm{B}}$ is the MTF of the linear image motion. The image motion was therefore extracted from the measured MTF. The flight parameters were the following:

$$
\begin{aligned}
& v / H=0.043421 / \mathrm{sec} \\
& T=1 / 700 \mathrm{sec} \\
& f^{\prime}=303.64 \mathrm{~mm}
\end{aligned}
$$

From equation (4) the image motion is obtained as

$$
\mathrm{a}=19 \mu \mathrm{m}
$$

Figure 3 (a) shows the MTF of image motion with $a=19 \mu \mathrm{m}$. In Figure 3 (b) $\mathrm{MTF}_{\mathrm{F}}, \mathrm{MTF}_{\mathrm{P}}$, and $\mathrm{MTF}_{\mathrm{B}} \cdot \mathrm{MTF}_{\mathrm{P}}$ are shown together. Good agreement was obtained between $\mathrm{MTF}_{\mathrm{F}}$ and $\mathrm{MTF}_{\mathrm{B}} \cdot \mathrm{MTF} \mathrm{F}_{\mathrm{P}}$.

Two airborne photographs of the same scene, taken with and without FMC, were compared to investigate the linear image motion. Corresponding roof-edges were chosen and scanned with the microdensitometer. From the parameters of the photograph $(\mathrm{v} / \mathrm{H}=$
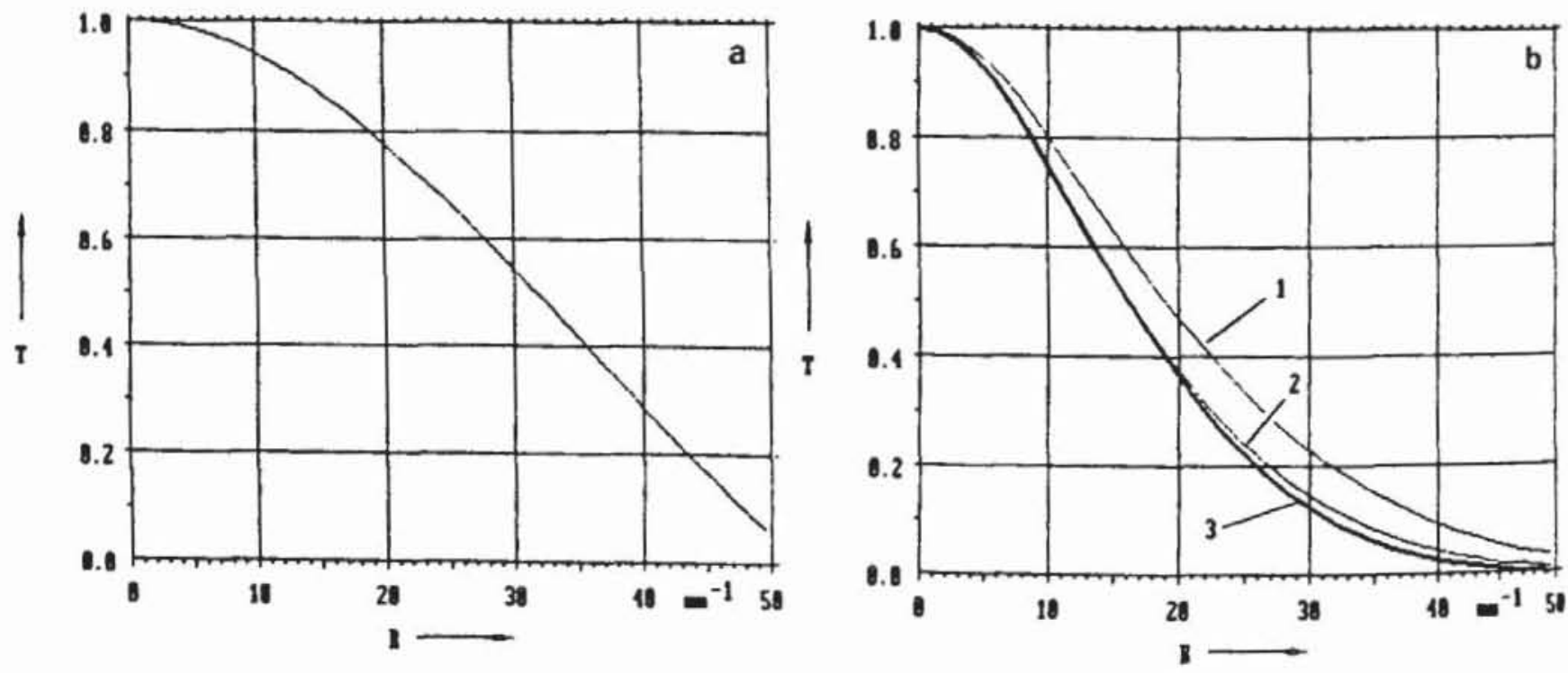

Fig.3 (a): MTF of an image motion with $a=19 \mu \mathrm{m} .(\mathrm{b}): \mathrm{MTF}_{\mathrm{F}}, \mathrm{MTF}_{\mathrm{P}}$, and $\mathrm{MTF}_{\mathrm{B}} \cdot \mathrm{MTF}_{\mathrm{P}} \cdot 1-\mathrm{MTF}_{\mathrm{P}}, 2-\mathrm{MTF}_{\mathrm{F}}, 3-\mathrm{MTF}_{\mathrm{B}} \cdot \mathrm{MTF} \mathrm{P}_{\mathrm{P}}$. 
$0.054911 / \mathrm{sec}, T=1 / 160 \mathrm{sec}$ and $\mathrm{f}^{\prime}=303.75 \mathrm{~mm}$ ) the image motion was calculated according to equation (4) to be $104 \mu \mathrm{m}$ as shown in Figure 4 (a). The MTF without FMC can be written as

$$
\mathrm{MTF}_{\mathrm{O}}=\mathrm{MTF}_{\mathrm{B}} \cdot \mathrm{MTF}_{\mathrm{C}}
$$

with $\mathrm{MTF}_{\mathrm{B}}$ as the MTF of the linear image motion and $\mathrm{MTF}_{\mathrm{C}}$ that of the airborne photograph with FMC. Figure 4 (b) shows the combined curves $\mathrm{MTF}_{\mathrm{O}}, \mathrm{MTF}_{\mathrm{C}}$, and $\mathrm{MTF}_{\mathrm{B}} \cdot \mathrm{MTF}_{\mathrm{C}}$. A major improvement of the image quality with FMC can be seen by comparing $\mathrm{MTF}_{O}$ and $\mathrm{MTF}_{\mathrm{C}}$. $\mathrm{MTF}_{\mathrm{O}}$ compares well with $\mathrm{MTF}_{\mathrm{B}} \cdot \mathrm{MTF}_{\mathrm{C}}$. One should notice that the first minimum and maximum of the sinc-function can be seen regarding the $\mathrm{MTF}_{\mathrm{O}}$.

It is shown that the use of FMC makes substantially better image quality possible. Aerial survey cameras with FMC allow the use of high-resolution, but unfortunately low-sensitivity films, making long exposure times necessary. In order to test the image quality of airborne photographs with different film types, the same scene was photographed with films with different sensitivity, using a camera with FMC. These airborne photographs were
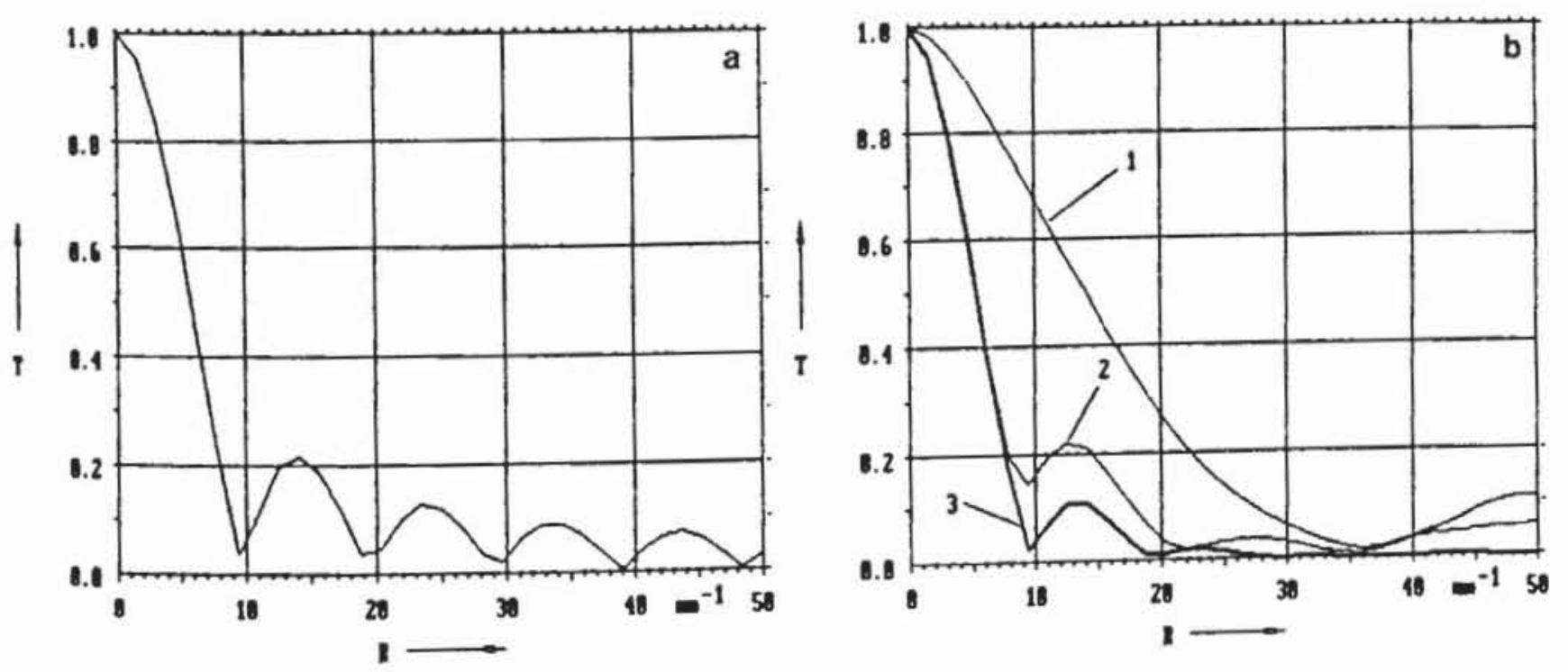

Fig. 4 (a): MTF of an image motion with $a=104 \mu \mathrm{m}$. (b): $\operatorname{MTF}_{O}$, MTF $_{C}$, and $\mathrm{MTF}_{\mathrm{B}} \cdot \mathrm{MTF}_{\mathrm{C}} \cdot 1-\mathrm{MTF}_{\mathrm{C}}, 2-\mathrm{MTF}_{\mathrm{O}}, 3-\mathrm{MTF}_{\mathrm{B}} \cdot \mathrm{MTF}_{\mathrm{C}}$. 
analysed by EGA. The measured results indicate that the finegrain, high-resolution emulsion did not lead to the expected best image quality. We found that the airborne photographs taken with longer exposure times were affected by vibration and roll of the aircraft during exposure.

\section{Study of the Transversal and Longitudinal object Motion at a close Range}

In close-range photogrammetry, the object motion reduces the image quality of the photographs, especially when the object is very close to the camera. The linear transversal motion of the object leads to a linear image motion, as mentioned above. A longitudinal object displacement results in a defocusing of the image plane.

In a laboratory experiment the influence on the image quality of the transversal and longitudinal object motion at close range were studied. A test target consisted of four edges. A pair of the edge patterns was oriented in the direction of the transversal object motion in order to determine experimentally the MTF of the lateral image motion, and another pair was oriented orthogonally to it. The target was placed into a precision $X-Y$-coordinate stage mounted on an optical bench and driven by computer controlled stepping motors. Using a camera, the test edges were photographed with the linear transversal object motion during exposure with and without a longitudinal object shift. The resulting edge images were scanned with a microdensitometer. The MTF's were obtained from this data using EGA.

A number of edge images having different amounts of image motion were investigated. The theoretical and experimental results agreed well.

A longitudinal focal shift arises from a longitudinal object shift. On the basis of geometrical optics, the defocused image point consists of a circular patch of light of radius $\Delta z^{\prime} \tan \alpha$ 
where $\alpha^{\prime}$ is the aperture angle in the image space and $\Delta z^{\prime}$ the defocusing. The defocused MTF is obtained by the Fourier-transform of the image patch. This is given by a Bessel function of first kind and first order

$$
\operatorname{MTF}_{\text {def }}=2 J_{2}(\mathrm{Z}) / \mathrm{Z}
$$

where $Z=2 \pi R \Delta z^{\prime} \tan \alpha^{\prime}$. The first zero of (8) occurs when $z=3.83$.

Figure 5(a) shows a defocused MTF curve calculated from the defocusing of $\Delta z^{\prime}=1.18 \mathrm{~mm}$, and corresponding longitudinal object shift of $10 \mathrm{~mm}$. Three curves are shown together in Figure $5(\mathrm{~b})$. Curves measured with and without defocusing 1, 2 respectively, illustrate the effect of the defocusing on the MTF measurement; Curve 3 was obtained by multiplying the MTF values measured in the proper focus by the known values of the defocused MTF. Good agreement was found with the MTF curve measured from the defocused photograph. From a set of defocused
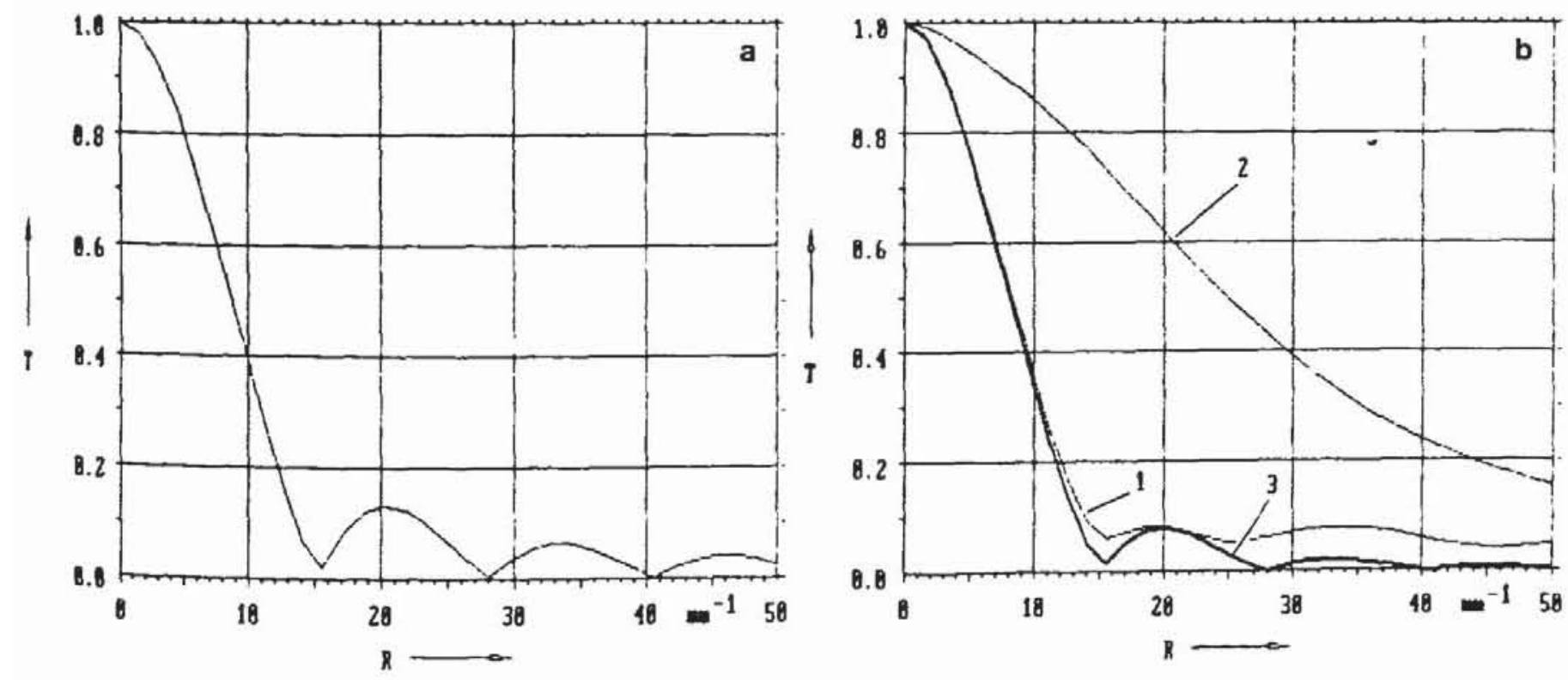

Fig. 5 (a): Defocused MTF curve with $\Delta z^{\prime}=1.18 \mathrm{~mm}$. (b): 1 - MTF curve measured with defocusing. 2 - MTF curve measured without defocusing. 3 - Calculated MTF curve with defocusing. 
MTF's, we found good agreement with the calculated values even for large amounts of defocusing. It was found that geometrical optics holds for large defects of focus.

\section{Conclusion}

Different methods to determine the MTF from image structures were discussed and compared. For EGA a new and relatively simple method was developed, applying a Hanning function. Alternatively a grating pattern in the same photograph was examined. The MTF's of both methods were found to be in good agreement. The two techniques can be used complementary and increase the reliability of image quality determination. EGA however has, from a practical point of view, the advantage not to rely on artificial edges, but to work almost as well on natural roof-edges. The resolution obtained from measured MTF's were compared with the resolution from three-bar targets. Again a good agreement was found. Furthermore, the influence of linear image motion was examined and the improvement of image quality with FMC discussed. For photographs taken with high resolution films, FMC is found to be necessary. Due to longer exposure times additional disturbance are introduced and need to be considered like vibrations. In laboratory measurements the image motion and the defocus at close range were examined. Even for large amounts of defocusing, the measurements agreed well with the calculations.

\section{Acknowledgement}

We would like to thank the DFG(SFB 228) for the financial support and the WILD corporation for the airborne photographs. 


\section{References}

/1/ Tiziani, H., 1977. Beurteilung der Bildqualität von Luftbildkammern: Vermessung, Photogrammetrie, Kulturtechnik. 4-77, pp. 137-146.

/2/ Scott, Frank, Scott, Roderic M., and Shack, Roland V., 1963. The Use of Edge Gradients in Determining ModulationTransfer Functions: Phot. Sci. Eng., Vol.7, No.6, pp. 345-349.

/3/ Jones, Robert A., 1967. An Automated Technique for Deriving MTF's from Edge Traces: Phot. Sci. Eng., Vol.11, No.2, pp. 102-106.

/4/ Jones, Robert A., and Yeadon, Edward C., 1969. Determination of the Spread Function from Noisy Edge Scans: Phot. Sci. Eng., Vol.13, No.4, pp. 200-204.

/5/ Sievers, J., 1976. Die Kantenbildanalyse als Mittel zur Bestimmung von "Bildparametern". Beitrag für die Arbeitsgruppe "semantische Information" der Kommission E der OEEPE.

/6/ Lei, Fang and Tiziani, H.J., 1986. Eine neue Methode zur Bestimmung der MTF aus dem Kantenbild: optik 74, No.1, pp. 17-21.

17/ Brigham, E. O., 1974. The Fast Fourier Transform. PrenticeHall, Inc. Englewood Cliffs, New Jersey.

/8/ Kölbl, O., 1985. Arbeitstagung: Kammerkalibrierung in der photogrammetrischen Praxis. Deutsche geodätische Kommission, Reihe B, Heft 275, S. 48-64. Verlag der Bayerischen Akademie der Wissenschaften, herausgegeben v. G. Kupfer u. W. Wester-Ebbinghaus. 